\title{
Extracellular vesicles in post-infarct ventricular remodelling
}

\author{
Aleksandra Gąsecka ${ }^{1,2}$, Edwin van der Pol ${ }^{2,3}$, Rienk Nieuwland², Ewa Stępień ${ }^{4}$ \\ ${ }^{1} 1^{\text {st }}$ Chair and Department of Cardiology, Medical University of Warsaw, Warsaw, Poland \\ ${ }^{2}$ Vesicle Observation Centre and Laboratory of Experimental Clinical Chemistry, Academic Medical Centre, \\ University of Amsterdam, Amsterdam, Netherlands \\ ${ }^{3}$ Biomedical Engineering and Physics, Academic Medical Centre, University of Amsterdam, Amsterdam, Netherlands \\ ${ }^{4}$ Marian Smoluchowski Institute of Physics, Jagiellonian University, Krakow, Poland
}

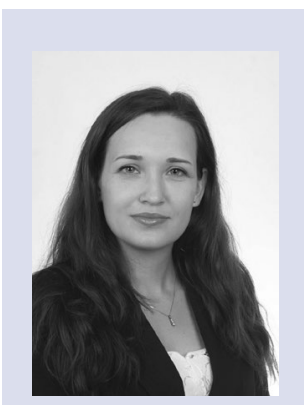

Aleksandra Gąsecka, MD is a cardiology resident and a $\mathrm{PhD}$ candidate in the $1^{\text {st }}$ Chair and Department of Cardiology, Medical University of Warsaw. She graduated with honours from the Medical University of Warsaw in 2015, received numerous scientific awards at international medical conferences, and completed several internships in Vienna, Zurich, and Amsterdam. Her scientific interests are novel biomarkers in cardiovascular disease, including extracellular vesicles, pharmacotherapy, and cardiovascular imaging. She is a Principal Investigator in the studies AFFECT EV (Antiplatelet therapy eFFECT on platelet Extracellular Vesicles in acute myocardial infarction) and PAPAYA (Platelet reactivity And treatment with Prostacyclin Analogues in pulmonarY Arterial hypertension), which are conducted in cooperation with Academic Medical Centre of the University of Amsterdam, The Netherlands and the European Health Centre Otwock, Poland. She is a member of the Young Thrombosis Researchers of the European Society of Cardiology.

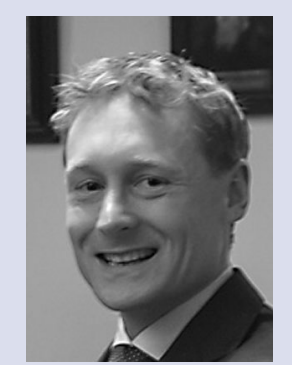

Edwin van der Pol, MSc, PhD, graduated in 2009 in Applied Physics from the University of Twente and from the institute for Atomic and Molecular Physics in Amsterdam. In 2015, he received his $\mathrm{PhD}$ cum laude on detection of extracellular vesicles. His $\mathrm{PhD}$ thesis was appreciated with a thesis award from the Dutch Society for Biophysics and Biomedical Engineering. He received numerous national and international scholarships, grants, and awards, including the prestigious Veni award from the Netherlands Organisation for Scientific Research, a keynote lecture at the Gordon Research Conference, and 18 invited lectures. To disseminate his inventions, Edwin co-founded the company Exometry (exometry.com). According to Google Scholar (August, 2017): 1741 citations, Hirsch index $=14$, i-10 index $=15$ (edwinvanderpol.com).

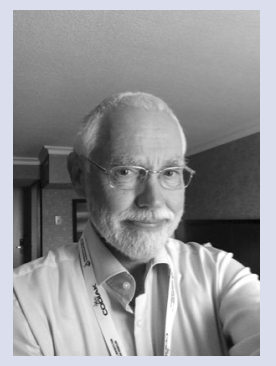

Doctor Rienk Nieuwland studied Biology in Utrecht and obtained his PhD from the Department of Haematology of the University Medical Centre Utrecht, the Netherlands. Since 2002, he has been working at the Academic Medical Centre of the University of Amsterdam, where he is Principal Investigator and Head of the Vesicle Observation Centre/Laboratory of Experimental Clinical Chemistry. His scientific interests are extracellular vesicles and platelets. He chairs the Scientific Standardisation Committee on Vascular Biology of the International Society on Thrombosis and Haemostasis, is a board member of the International Society on Extracellular Vesicles, vice-president of the Dutch Society on Extracellular Vesicles, a coordinator of the European Metrology Research Project METVES (www.metves.eu), and one of the founders of the CANCER-ID project (started 2015). He is a co-author of over 160 papers indexed in PubMed MEDLINE; according to Google Scholar database (August, 2017): 11,432 citations, Hirsch index $=55$, i-10 index $=113$.

\section{Address for correspondence:}

Aleksandra Gąsecka, $1^{\text {st }}$ Chair and Department of Cardiology, Medical University of Warsaw, ul. Banacha 1A, 02-097 Warszawa, Poland,

e-mail: aleksandra.gasecka@wum.edu.pl

Received: 06.09.2017 Accepted: 06.09.2017 Available as AoP: 13.09.2017

Kardiologia Polska Copyright (C) Polskie Towarzystwo Kardiologiczne 2018 


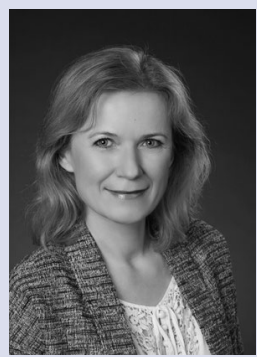

Ewa Stępień, Associate Professor, is a molecular biologist with medical specialty in clinical genetics and the Head of the Department of Medical Physics at Marian Smoluchowski Institute of Physics, Jagiellonian University in Krakow, Poland. Her main fields of interests cover the area of new biomarkers in cardiovascular disease, with a focus on extracellular vesicles and microRNA as potential targets in diagnostics and therapy. As a group leader, she conducts and supervises a number of projects dedicated to the role of extracellular vesicles in endothelial dysfunction and angiogenesis. As an Associate Professor, she gives courses in cell biology and clinical biochemistry for students in medical physics. She is a member of the Experimental Cardiology Section of the Polish Cardiac Society and a member of the Working Group on Atherosclerosis and Vascular Biology and the Working Group on Thrombosis of the European Society of Cardiology. She is also a member of the International Society of Extracellular Vesicles.

\section{INTRODUCTION}

Heart failure (HF) is a major public health problem, with a global prevalence of $1-2 \%$ of the adult population, rising to $10 \%$ among people over 70 years of age [1]. The economic costs of HF to healthcare systems and society are estimated at $\$ 108$ billion per year [2]. As the population ages and the survival rate of cardiovascular disease increases due to improved treatment, the number of patients with HF is expected to grow [3]. Despite recent progress in pharmacological treatment, mechanical circulatory support, and multidisciplinary team management, the prognosis of patients with HF is still poor, with up to $50 \%$ of the patients dying within five years of the diagnosis [4].

Acute myocardial infarction (AMI) causes over $50 \%$ of cases of HF, followed by arterial hypertension, valvular heart disease, cardiomyopathies, and other causes [5]. In patients with $\mathrm{AMI}$, the myocardial necrosis leads to replacement of the infarcted area by scar tissue. These structural and functional changes of the myocardium are termed post-infarct left ventricle remodelling (LVR), which leads to the loss of contractile tissue and subsequently to post-infarct HF [6]. LVR is defined as an increase in end-diastolic left ventricular volume by $20 \%$ at six months after AMI, often accompanied by development of functional mitral regurgitation [6]. LVR affects $30 \%$ of patients after $\mathrm{AMI}$, deteriorating the prognosis [7]. Clinical, biochemical, and imaging parameters predictive of LVR after AMI include the extension and anterior location of AMI [8], late or unsuccessful revascularisation [8], high concentrations of natriuretic peptides and cardiac troponins [9], enlarged left ventricular chamber on echocardiography [10], and microvascular obstruction and/or intramyocardial haemorrhage on cardiac magnetic resonance imaging [10]. However, none of these parameters predict which patients will develop LVR and progress to HF.

The pathways promoting development of LVR are (i) chronic stimulation of beta-adrenergic receptors and (ii) activation of the renin-angiotensin-aldosterone system (RAAS). Therefore, drugs that inhibit these pathways, including beta-blockers, angiotensin-converting enzyme inhibitors, angiotensin receptor blockers, and aldosterone antagonists, are established to prevent LVR in patients after AMI [11]. Furthermore, mechanical interventions, such as cardiac resynchronisation therapy and left ventricle assist devices, partly reverse remodelling in eligible subsets of patients with advanced HF [4]. Finally, the intramyocardial administration of pluripotent stem cells improves ventricular function in patients with HF [12]. Nevertheless, none of the available treatments entirely prevent, inhibit, or reverse LVR. Thus, post-infarct LVR lacks both reliable prediction and effective treatment.

Extracellular vesicles (EVs) are biological nanoparticles with a phospholipid bilayer probably released by all eukaryotic and prokaryotic cells to the extracellular environment. Consequently, body fluids such as blood, urine, and probably pericardial fluid contain EVs [13, 14]. A transmission electron microscope (TEM) image and a cryo-TEM image of EVs from human plasma are shown in Figure 1. EVs are capable of transferring proteins, nucleic acids, and signalling ligands between cells, by which they affect the recipient cells [13]. Evidence is accumulating that EVs released from cardiac cells mediate the complex interplay between cardiomyocytes (CMs), fibroblasts, endothelial cells, vascular smooth muscle cells, and extracellular matrix underlying LVR [15]. Depending on the cellular origin and concentration of EVs, EVs are either cardioprotective or promote adverse LVR [16]. Hence, EVs from cardiac cells are candidate biomarkers to predict LVR, and are potential drug vehicles of LVR therapy.

Here we present the historical background of EVs, introduce EVs derived from cardiac cells (section 1), summarise the current evidence on the role of EVs in cardioprotection (section 2) and adverse post-infarct LVR (section 3), and present the potential clinical applications of EV-based biomarkers and therapeutics (section 4). The structure of the review is shown in Figure 2.

\section{HISTORY OF EXTRACELLULAR VESICLES}

Extracellular vesicles were identified in 1967 as a subcellular fraction of plasma called "platelet dust", because this fraction was thought to originate from platelets only [17]. Subsequently, 

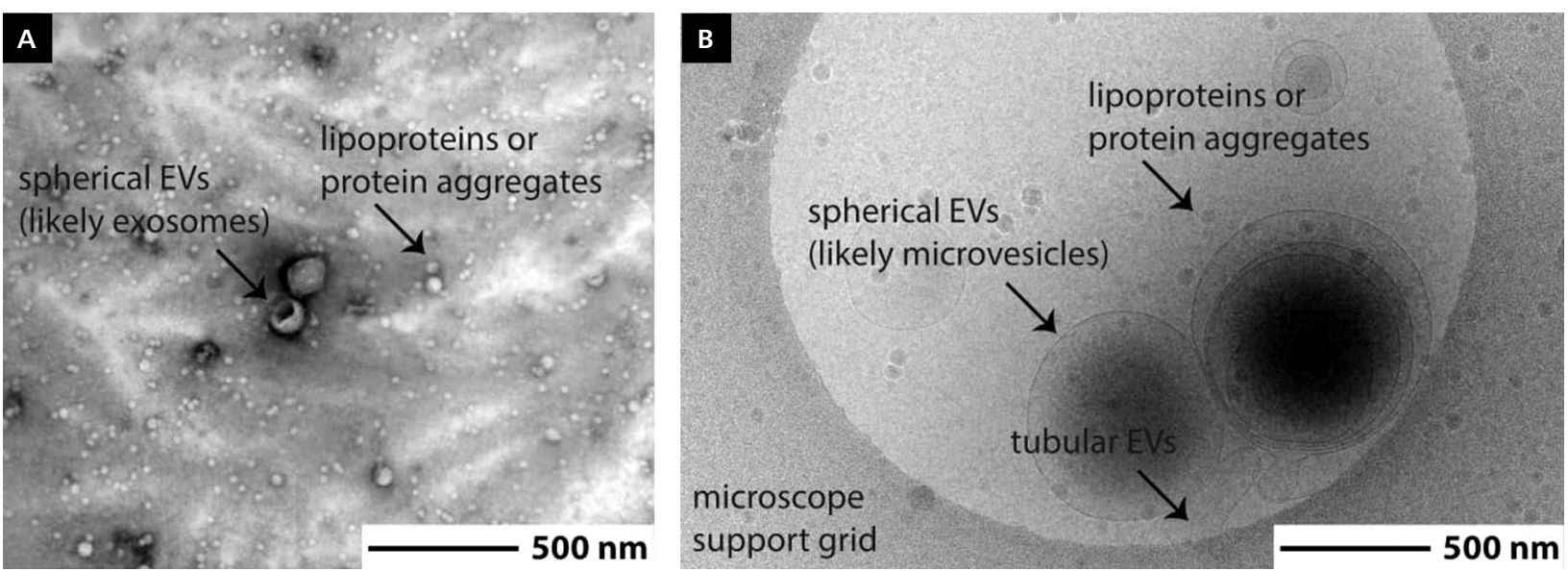

Figure 1. A transmission electron microscope (TEM) image (A) and a cryo-TEM image (B) of extracellular vesicles (EVs) from human plasma. The black arrows point to small spherical EVs, probably exosomes, large multi-layered EVs, probably microvesicles, and tubular EVs. The black arrows point also to lipoproteins or protein aggregates, which could be interpreted as small EVs. Image courtesy of Linda G. Rikkert, Vesicle Observation Centre, Academic Medical Centre, University of Amsterdam, the Netherlands and M. Libera, Centre of Polymer and Carbon Materials, Polish Academy of Sciences, Zabrze, Poland

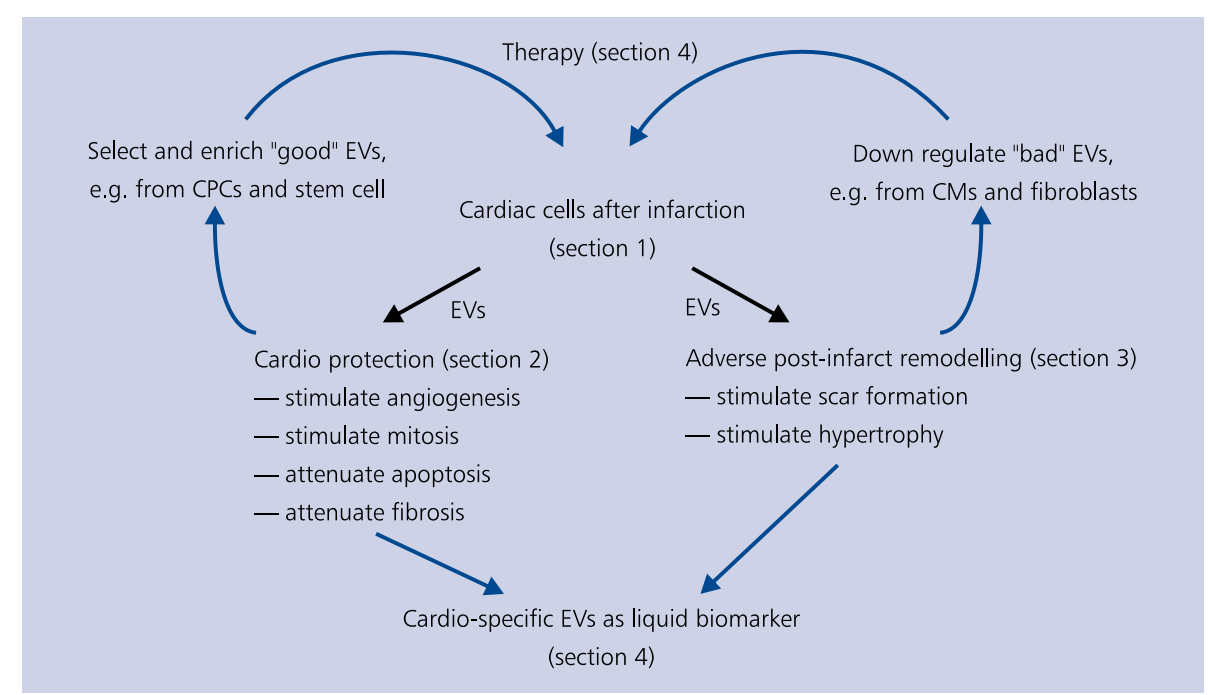

Figure 2. The structure of the review. After acute myocardial infarction, cardiac cells release extracellular vesicles (EVs), which either contribute to cardioprotection or to adverse post-infarct remodelling. Analysis of cardio-specific EVs in blood can be used to predict and/or diagnose early post-infarct remodelling. Selection and enrichment of EVs from cardiac progenitor cells (CPCs), stem cells, cardiomyocytes (CMs), and fibroblasts can be used for therapy

also the occurrence of EVs in plasma, that were derived from cells other than platelets was demonstrated, and EVs with platelet features were shown to be released by platelet precursor cells, megakaryocytes [18, 19]. At present, EVs from platelets and/or megakaryocytes are thought to constitute about $30 \%$ of all circulating EVs [20], whereas the remaining EVs originate from blood cells, endothelial cells, and probably tissues, including the myocardium [21].

Initially, EVs were named after the cells or tissues from which they originate, e.g. prostasomes for EVs from prostate and dexosomes for EVs from dendritic cells, regardless of their bio- physical and biochemical features, which caused confusion in the nomenclature [13]. This confusion was further increased by the historical and artificial division of EV into "exosomes", "mic rovesicles"/"microparticles", and "apoptotic bodies", based on the theoretical differences in size, mechanism of biogenesis, and exposed protein markers [13]. Along with the development of sensitive detection techniques, it has become clear that at present no biophysical or biochemical feature distinguishes types of EVs from each other, and that EVs in human body fluids are more heterogeneous than EVs from in vitro sources, like cell cultures. Therefore, the umbrella term "extracellular vesicles" 


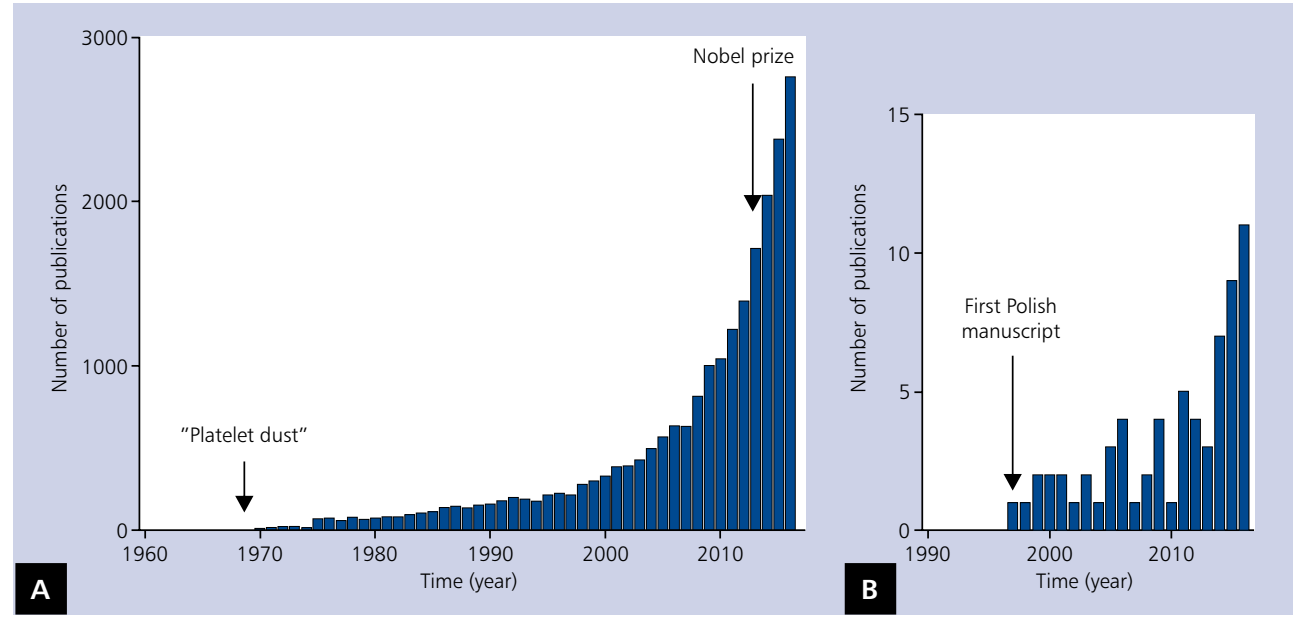

Figure 3. The number of global (A) and Polish (B) annual publications on extracellular vesicles (EVs) versus time, with an extensive increase in the last decade. In the least 50 years EVs have made their way from "platelet dust" into mediators of intercellular communication, the discovery of which was awarded the Nobel Prize in Physiology and Medicine

has been recommended to facilitate exchange of information within the EV research community [22].

Nearly five decades of research on EVs have made their way from cell debris into mediators of intercellular communication, which was awarded the Nobel Prize in Physiology or Medicine on the machinery regulating vesicle traffic within a cell, awarded to Randy Schekman et al. in 2013. It has been recognised that $\mathrm{EV}$ s have other functions beyond coagulation, such as inflammation [23] and remodelling of the infarcted myocardium [15]. Additionally, because the concentration of EVs probably ranges between $10^{7}$ and $10^{9} \mathrm{EVs} / \mathrm{mL}$ in the plasma of healthy individuals, which is comparable to the concentration of platelets or red blood cells [22], and because EVs transport proteins, nucleic acids, and signalling ligands between the cells, EVs are likely to contribute to physiological processes such as haemostasis [24], immune response [25], and cardioprotection [26]. Because EVs (i) expose specific receptors or ligands to target the recipient cells, (ii) have a unique protein/nucleic acid composition transferred to recipient cells, (iii), have a rigid lipid membrane to protect their cargo from enzymatic degradation, and (iv) have biophysical properties that enable separation from lipoproteins and protein aggregates, EVs have potential clinical applications as diagnostic/prognostic biomarkers, and as autologous vehicles for drug delivery. As a result, the clinical and scientific interest in EVs has grown tremendously. Figure 3 shows that the number of scientific publications on EVs versus time is growing exponentially. To establish EVs as clinically relevant biomarkers, standardised methodology to analyse EVs is required. Along with the "Guidelines to study extracellular vesicles" [27] and recent technological improvements, the field of EVs is entering an era of clinical application [22]. One of the promising applications of EVs is the prediction and treatment of post-infarct LVR.

\section{BIOGENESIS, CARGO, AND FUNCTION OF CARDIAC-DERIVED EXTRACELLULAR VESICLES (SECTION 1)}

After AMI, the cardiac cells including CMs, fibroblasts, endothelial cells, vascular smooth muscle cells, immune cells, and cardiac progenitor cells (CPCs) communicate with each other and recruit stem cells from the bone marrow to induce repair of the infarcted myocardium (Fig. 2, section 1) [28]. This adaptive repair is orchestrated by different signalling molecules, including cytokines and growth factors secreted from cardiac cells, which may be transported by EVs, at least in vitro [29].

It was demonstrated that highly differentiated cultured CMs release EVs ranging from 40 to $300 \mathrm{~nm}$ in diameter [29]. It was shown that CM-derived EVs contain cardiac-specific sarcomeric and mitochondrial proteins, including tropomyosin, myomesin, and cardiac-type myosin-binding protein $\mathrm{C}$ [30]. Moreover, under ischaemic conditions CMs release EVs containing proteins that cause inflammation (heat shock proteins), CM apoptosis (tumour necrosis factor alpha), hypertrophy, and fibrosis (transforming growth factor [TGF]-beta and transglutaminase 2) [30-32]. Hence, the milieu of the cardiac cells influences the composition and quantity of EVs released to the extracellular environment, suggesting that circulating EVs may represent a snapshot of the pathophysiological state of the myocardium.

Soon after the proteome of CM-derived EVs had been determined, another landmark finding followed. CM-derived EVs were shown to contain over 1500 different mRNA transcripts 
and 340 distinct DNA sequences, which were taken up by fibroblasts and reprogrammed gene expression in fibroblasts [32]. Additionally, it has been proposed that EVs contribute to intercellular transfer of microRNAs (miRNAs) [33]. MiRNAs are highly conserved, about 22 nucleotides long, noncoding RNA molecules. By binding to complementary sequences on target mRNAs, miRNAs regulate the mRNA production, thereby affecting cell proliferation, differentiation, and apoptosis [33].

\section{EXTRACELLULAR VESICLES IN CARDIOPROTECTION (SECTION 2)}

Cardioprotection is defined as "all mechanisms and means that contribute to the preservation of the heart by preventing or reversing myocardial damage" [26]. Because CMs are terminally differentiated, reversing myocardial damage is feasible only by recruitment of resident CPCs and non-cardiac progenitor and/or stem cells to the infarcted myocardium. Consequently, the direct administration of stem cells was thought to improve the cardiac repair after AMI by the replacement of damaged CMs with new cells [34]. Whereas the stem cells injected into the coronary circulation poorly differentiated into CMs, improvement of cardiac function and survival was consistently observed [34]. Subsequently, the stem cell-derived paracrine factors, including EVs and EV-derived miRNAs, have been implicated in cardioprotective or therapeutic activities of pluripotent stem cells (Fig. 2, section 2) [35, 36]. EVs purified from the conditioned medium of CPCs were shown to be enriched in proangiogenic miR-210, miR-132, and miR-146a, which stimulated proliferation and migration of human umbilical vein endothelial cells, and increased the survival of CMs in cell culture [36]. Of note, EVs from CPCs cultured in hypoxic conditions contained higher amounts of proangiogenic miR132 and miR-146a compared to EVs released from CPCs under normoxia [36], suggesting that hypoxia probably increases the proangiogenic potential of CPC-derived-EVs also in vivo, for example in patients with atherosclerosis and/or HF. Research is ongoing to manipulate stem cells to release EVs containing specific proteins for delivery [37]. For example, the CD34 ${ }^{+}$ stem cells engineered to release EVs containing the morphogen sonic hedgehog injected into the border infarct zone of mice reduced the infarct size, increased capillary density, and improved long-term functional recovery [37]. EVs from CD34+ stem cells were selectively taken up by CMs and cardiac endothelial cells, but not by fibroblasts, suggesting the presence of cell-specific receptors on EVs from CD34+ stem cells [37]. If so, the cardioprotective and regenerative properties of EVs derived from cardiac and non-cardiac progenitors and/or stem cells might be used in cell-free therapy to augment regeneration of the post-infarct myocardium by activating canonical pathways involved in de-differentiation and re-differentiation processes [33, 35, 36]. One should bear in mind, however, that some effects initially attributed to EVs are caused by co-isolation contaminants. For example, the part of miRNAs previously thought to be present in and transported by EVs is now known to be transported by high-density lipoproteins and/or argonaute-2 protein, which co-isolate with EVs upon centrifugation [22].

Cardiac cells surviving in the infarcted myocardium prevent myocardial damage by release of EVs enriched with pro-angiogenic, mitogenic, anti-apoptotic, and anti-fibrotic factors, thereby inducing metabolic and structural adaptations. The growth of new vessels, neoangiogenesis, is a major adaptation after $\mathrm{AMI}$, allowing redistribution of the cardiac blood supply from the necrotic to the viable myocardium. The main trigger for neoangiogenesis is ischaemic preconditioning, defined as protecting the heart against permanent injury after alternate periods of myocardial ischaemia and reperfusion [38]. EVs were demonstrated to mediate ischaemic preconditioning by changing the expression of specific miRNAs. For example, miR-21 and miR-126 in EVs from endothelial cells increased the resistance of CMs to hypoxic stress by stimulation of phosphoinositide 3-kinase/protein kinase B and other pro-survival pathways [39]. Moreover, miR-21 regulates the expression of extracellular matrix metalloproteinase (MMPs) inducer and MMPs responsible for the remodelling of extracellular matrix, thereby facilitating the migration of endothelial cells and neoangiogenesis [40]. Besides miRNAs, growth factors including insulin-like growth factor 1, TGF-beta, and vascular endothelial growth factor. Also, heat shock proteins are present in EVs from cardiac stem cells, and these proteins can be transferred to adjacent cells to stimulate proliferation and confer protection against oxidative stress, respectively [30, 41]. Finally, EVs from CMs are enriched in miR-29b and miR-133a, which both suppress cardiac fibrosis by targeting connective tissue growth factor, type 1 collagen, and TGF-beta $[42,43]$. Again, the effects attributed to EVs should be interpreted with caution, until revised with use of novel methods to isolate and concentrate EVs.

\section{EXTRACELLULAR VESICLES IN ADVERSE \\ POST-INFARCT REMODELLING (SECTION 3)}

The main processes in post-infarct LVR are scar formation within the infarcted wall and hypertrophy of the remaining walls [28].

Scar formation involves (i) remodelling of the extracellular matrix, (ii) transformation of cardiac fibroblasts to myofibroblasts, and (iii) transformation of endothelial cells to mesenchymal stem cells, termed endothelial-to-mesenchymal transition (EMT). EVs contribute to these processes (Fig. 2, section 3) [15, 44, 45].

First, neutrophils and macrophages which migrate into the infarcted zone release EVs containing miR-21 and miR-26a, which both are responsible for the remodelling of the extracellular matrix by regulating the expression of MMPs, such as MMP-2, -3, -7, and -13 [44]. MMPs digest the infarcted tissue, thereby facilitating the invasion of myofibroblasts and stimu- 
lating the migration of inflammatory cells. Myofibroblasts, in turn, produce collagen, which is deposited in the infarcted myocardium to form connective tissue. Myofibroblasts also release EVs containing TGF-beta, which induces EMT and fibrosis [30, 45]. Following EMT, endothelial cells increase the synthesis of extracellular matrix components. As a result, the infarcted tissue is progressively replaced by a scar. Of note, the contribution of EVs to scar formation has been investigated in vitro, leaving the actual role of EVs in scar formation in vivo not established.

Hypertrophy is initiated by (i) local and systemic tissue synthesis of RAAS components, angiotensin II, and aldosterone, (ii) enhanced release of catecholamines by adrenal medulla, and (iii) the release of paracrine/autocrine factors, such as miRNAs [28].

Stimulation with angiotensin II increases the release of EVs from cultured cardiac fibroblasts [46]. The released EVs enhanced the production of angiotensin II and increased the expression of angiotensin II receptor type 1 and 2 in CMs, thereby inducing pathological hypertrophy of CMs [46]. The EV-induced hypertrophy of CMs was abolished in the presence of the EV inhibitors GW4869 and dimethyl amiloride, further confirming that pathological cardiac hypertrophy induced by activation of RAAS is - at least partly - mediated by fibroblast-derived EVs [46]. The effect of catecholamines on the release of EVs from cardiac cells has not been studied so far, but in a murine model EVs from hepatocytes contain catechol O-methyltransferase (COMT), which inactivates dopamine, epinephrine, and norepinephrine. Thus, release of these hepatocyte-derived EVs into the circulation may be involved in the metabolism of catecholamines [47]. By changing the quality and quantity of catecholamines that bind to the cardiac alpha- and beta-adrenergic receptors, COMT-containing EVs may affect the growth and proliferation of CMs. Hence, modulation of COMT activity in circulating EVs offers new possibilities to decrease the concentration of catecholamines in patients after AMI, thereby decreasing the risk of LVR and HF.

Among the paracrine factors essential for hypertrophy, EV-mediated transfer of miRNAs between the cardiac muscle cells plays a central role. Cardiac fibroblasts release EVs enriched in miR-21, which induces hypertrophy of CMs by downregulating the expression of proteins involved in organising cardiac muscle structure and function, such as sorbin and $\mathrm{SH} 3$ domain-containing protein 2 [48]. In a murine model, inhibition of miR-21 suppressed CM hypertrophy in response to angiotensin II, thus confirming in vivo that fibroblast-derived miR-21 is involved in CM hypertrophy [49]. Other EV-derived miRNAs released from cardiac fibroblasts, which are involved in hypertrophy, include miR-133a, miR-208, and miR499. These miRNAs regulate genes encoding calcium channel inositol trisphosphate 3 receptor gene and myosin heavy chain [49]. Whereas in physiological conditions miR-133a, miR-
208, and miR-499 stimulate heart healing, overproduction of these miRNAs during LVR favours the development of cardiac hypertrophy [49]. Probably the same miRNAs have either an adaptive or maladaptive role because they activate different intracellular pathways, depending on their concentration and/or duration of stress conditions. Hence, miRNA-regulated intracellular pathways might become a novel therapeutic tool for prevention and treatment of LVR.

\section{CONCLUSIONS AND CLINICAL IMPLICATIONS (SECTION 4)}

Identification of EV subtypes specific for LVR may be a novel source of biomarkers with the potential to identify patients at risk of LVR and HF (Fig. 2, section 4). Moreover, understanding the mechanisms involved in the release and uptake of EVs creates new opportunities to control and govern LVR by targeted delivery of EVs containing cardioprotective drugs (Fig. 2, section 4). Of note, EV-mediated therapy opens up a new treatment strategy by using stem cell implantation in the replacement of damaged CMs and restitution of the infarcted myocardium. However, before the results of the in vitro and the preliminary in vivo studies in animal models are translated into clinical practice, some issues need to be clarified.

First, a typical primary CM culture is not homogenous, and therefore it is challenging to determine whether EVs secreted to the conditioned media in the culture of CMs are indeed released from the CMs or from contaminating cells such as fibroblasts and/or endothelial cells. Second, the secretory potential of a cell line is usually different in in vitro than in in vivo conditions, which warrants caution while translating the experimental results to the clinical models. Third, EVs transport a number of still unidentified proteins and nucleic acids, so it is difficult to attribute a specific effect evoked by EVs to a specific molecule. Finally, it seems that EVs from the same cell line have dual activity, being either pro- or antiangiogenic, pro- or antifibrotic, mitogenic or apoptotic, depending on their intracellular origin and concentration, thus making it difficult to predict the biological effects after administration to patients [32, 33, 50]. Altogether, the biosafety and efficacy of EV-based therapy in LVR and other cardiovascular disease should be carefully assessed in preclinical studies before moving this promising field towards clinical trials.

In an attempt to standardise the methodology of EV analysis and to accelerate the clinical implementation of EVs, the international research community, including Polish researchers, has joined forces to create the International Society of Extracellular Vesicles (ISEV). Figure 4 shows an overview of the Polish institutions that use the three most common technologies to characterise single EVs: EV-dedicated flow cytometry capable of analysing EVs > $150 \mathrm{~nm}$, nanoparticle tracking analysis (NTA), and tuneable resistive pulse sensing (TRPS). Figure 4 shows that there are five EV-dedicated flow cytometers (Apogee A50 or A60), four TRPS, and one NTA 


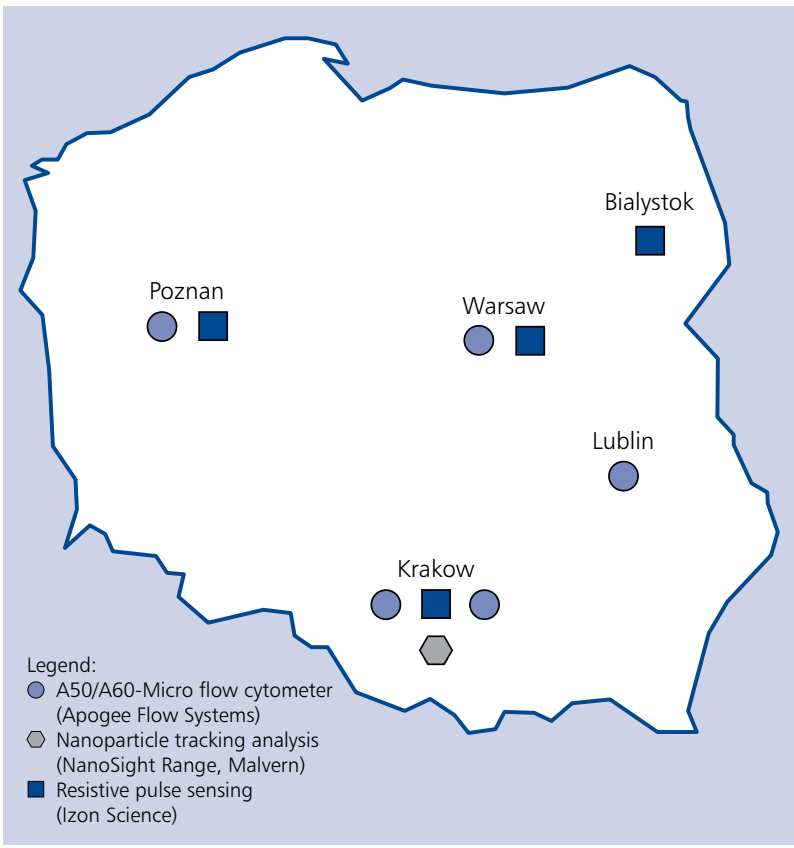

Figure 4. Overview of the institutions that use the three most common technologies (dedicated flow cytometry, nanoparticle tracking analysis, and resistive pulse sensing) to characterise single extracellular vesicles in Poland

in Poland. Regarding the substantial scientific potential of the individual institutions, which could be augmented by the inter-institutional collaboration, attempts are ongoing to launch the Polish Society of EVs, the main goal of which would be to facilitate the exchange of information and technologies between the institutions and promote $\mathrm{EV}$ research at funding agencies, thereby contributing to the worldwide efforts to improve healthcare by EVs.

To conclude, the EV field is new and the roles of EVs in health and disease are undergoing extensive research. It is tempting to speculate that EVs might be used as liquid biomarkers to predict LVR, and/or as drug vehicles of LVR therapy. However, before EVs are clinically applicable, standardisation of the pre-analytical and analytical variables is required based on the detection of EVs by sensitive analytical techniques. Recent progress regarding the isolation and detection of EVs, as well as new insights into their concentration and function, has allowed the establishment of the first consensus document in the field: "Methodological guidelines to study extracellular vesicles" [27]. To achieve the consensus, the interdisciplinary collaboration between researchers from various disciplines and societies is much needed. Following the consensus, the field of EVs is currently entering the era of clinical studies.

Conflict of interest: none declared

\section{References}

1. Roger VL. Epidemiology of heart failure. Circ Res. 2013; 113(6): 646-659, doi: 10.1161/CIRCRESAHA.113.300268, indexed in Pubmed: 23989710.

2. Cook C, Cole G, Asaria P, et al. The annual global economic burden of heart failure. Int J Cardiol. 2014; 171(3): 368-376, doi: 10.1016/j. ijcard.2013.12.028, indexed in Pubmed: 24398230.

3. Mathers CD, Loncar D. Projections of global mortality and burden of disease from 2002 to 2030. PLoS Med. 2006; 3(11): e442, doi: 10.1371/journal.pmed.0030442, indexed in Pubmed: 17132052.

4. Taylor CJ, Roalfe AK, Iles R, et al. Prognosis of all-cause heart failure and borderline left ventricular systolic dysfunction: 5 year mortality follow-up of the Echocardiographic Heart of England Screening Study (ECHOES). Eur Heart J. 2007; 28(9): 1128-1134, doi: 10.1093/eurheartj/ehm102, indexed in Pubmed: 17459902.

5. Fox KF, Cowie MR, Wood DA, et al. Coronary artery disease as the cause of incident heart failure in the population. Eur Heart J. 2001; 22(3): 228-236, doi: 10.1053/euhj.2000.2289, indexed in Pubmed: 11161934.

6. Yano T, Miura T, Ikeda Y, et al. Intracardiac fibroblasts, but not bone marrow derived cells, are the origin of myofibroblasts in myocardial infarct repair. Cardiovasc Pathol. 2005; 14(5): 241-246, doi: 10.1016/j. carpath.2005.05.004, indexed in Pubmed: 16168896.

7. Flachskampf FA, Schmid M, Rost C, et al. Cardiac imaging after myocardial infarction. Eur Heart J. 2011; 32(3): 272-283, doi: 10.1093/eurheartj/ehq446, indexed in Pubmed: 21163851.

8. Orn S, Manhenke C, Anand IS, et al. Effect of left ventricular scar size, location, and transmurality on left ventricular remodeling with healed myocardial infarction. Am J Cardiol. 2007; 99(8): 1109-1114, doi: 10.1016/j.amjcard.2006.11.059, indexed in Pubmed: 17437737.

9. Hallén J, Jensen JK, Fagerland MW, et al. Cardiac troponin I for the prediction of functional recovery and left ventricular remodelling following primary percutaneous coronary intervention for ST-elevation myocardial infarction. Heart. 2010; 96(23): 1892-1897, doi: 10.1136/hrt.2009.190819, indexed in Pubmed: 21062778.

10. Husser O, Monmeneu JV, Sanchis J, et al. Cardiovascular magnetic resonance-derived intramyocardial hemorrhage after STEMI: Influence on long-term prognosis, adverse left ventricular remodeling and relationship with microvascular obstruction. Int J Cardiol. 2013; 167(5): 2047-2054, doi: 10.1016/j.ijcard.2012.05.055, indexed in Pubmed: 22682700.

11. Frigerio M, Roubina E. Drugs for left ventricular remodeling in heart failure. Am J Cardiol. 2005; 96(12A): 10L-18L, doi: 10.1016/j. amjcard.2005.09.060, indexed in Pubmed: 16399088.

12. Gwizdala A, Rozwadowska N, Kolanowski TJ, et al. Safety, feasibility and effectiveness of first in-human administration of muscle-derived stem/progenitor cells modified with connexin-43 gene for treatment of advanced chronic heart failure. Eur J Heart Fail. 2017; 19(1): 148-157, doi: 10.1002/ejhf.700, indexed in Pubmed: 28052545.

13. van der Pol E, Böing AN, Harrison P, et al. Classification, functions, and clinical relevance of extracellular vesicles. Pharmacol Rev. 2012; 64(3): 676-705, doi: 10.1124/pr.112.005983, indexed in Pubmed: 22722893

14. Beltrami C, Besnier M, Shantikumar S, et al. Human pericardial fluid contains exosomes enriched with cardiovascular-expressed micrornas and promotes therapeutic angiogenesis. Mol Ther. 2017; 25(3): 679-693, doi: 10.1016/j.ymthe.2016.12.022, indexed in Pubmed: 28159509.

15. Waldenström A, Ronquist G. Role of exosomes in myocardial remodeling. Circ Res. 2014; 114(2): 315-324, doi: 10.1161/CIRCRESAHA.114.300584, indexed in Pubmed: 24436427.

16. Ailawadi $\mathrm{S}$, Wang $\mathrm{X}, \mathrm{Gu} \mathrm{H}$, et al. Pathologic function and therapeutic potential of exosomes in cardiovascular disease. Biochim Biophys Acta. 2015; 1852(1): 1-11, doi: 10.1016/j.bbadis.2014.10.008, indexed in Pubmed: 25463630.

17. Wolf $P$. The nature and significance of platelet products in human plasma. Br J Haematol. 1967; 13(3): 269-288, indexed in Pubmed: 6025241

18. Flaumenhaft R, Dilks JR, Richardson J, et al. Megakaryocyte-derived microparticles: direct visualization and distinction from platelet-derived microparticles. Blood. 2009; 113(5): 1112-1121, doi: 10.1182/blood-2008-06-163832, indexed in Pubmed: 18802008. 
19. Rank A, Nieuwland R, Delker R, et al. Cellular origin of platelet-derived microparticles in vivo. Thromb Res. 2010; 126(4): e255-e259, doi: 10.1016/j.thromres.2010.07.012, indexed in Pubmed: 20696467.

20. Arraud N, Linares R, Tan S, et al. Extracellular vesicles from blood plasma: determination of their morphology, size, phenotype and concentration. J Thromb Haemost. 2014; 12(5): 614-627, doi: 10.1111/jth.12554, indexed in Pubmed: 24618123.

21. Malik ZA, Kott KS, Poe AJ, et al. Cardiac myocyte exosomes: stability, HSP60, and proteomics. Am J Physiol Heart Circ Physiol. 2013 304(7): H954-H965, doi: 10.1152/ajpheart.00835.2012, indexed in Pubmed: 23376832.

22. van der Pol E, Böing AN, Gool EL, et al. Recent developments in the nomenclature, presence, isolation, detection and clinical impact of extracellular vesicles. J Thromb Haemost. 2016; 14(1): 48-56, doi: 10.1111/jth.13190, indexed in Pubmed: 26564379.

23. Vajen T, Mause SF, Koenen RR. Microvesicles from platelets: novel drivers of vascular inflammation. Thromb Haemost. 2015; 114(2): 228-236, doi: 10.1160/TH14-11-0962, indexed in Pubmed: 25994053.

24. Gasecka A, Böing AN, Filipiak KJ, et al. Platelet extracellular vesicles as biomarkers for arterial thrombosis. Platelets. 2017; 28(3): 228-234, doi: 10.1080/09537104.2016.1254174, indexed in Pubmed: 27996341.

25. Robbins PD, Morelli AE. Regulation of immune responses by extracellular vesicles. Nat Rev Immunol. 2014; 14(3): 195-208, doi: 10.1038/nri3622, indexed in Pubmed: 24566916.

26. Yellon DM, Davidson SM. Exosomes: nanoparticles involved in cardioprotection? Circ Res. 2014; 114(2): 325-332, doi: 10.1161/CIRCRESAHA.113.300636, indexed in Pubmed: 24436428.

27. Coumans FAW, Brisson AR, Buzas EI, et al. Methodological Guidelines to Study Extracellular Vesicles. Circ Res. 2017; 120(10) 1632-1648, doi: 10.1161/CIRCRESAHA.117.309417, indexed in Pubmed: 28495994

28. Sutton MG, Sharpe N. Left ventricular remodeling after myocardial infarction: pathophysiology and therapy. Circulation. 2000; 101(25): 2981-2988, indexed in Pubmed: 10869273.

29. Gupta S, Knowlton AA. HSP60 trafficking in adult cardiac myocytes role of the exosomal pathway. Am J Physiol Heart Circ Physiol. 2007; 292(6): H3052-H3056, doi: 10.1152/ajpheart.01355.2006, indexed in Pubmed: 17307989.

30. Borges FT, Melo SA, Özdemir BC, et al. TGF- $\beta$ 1-containing exosomes from injured epithelial cells activate fibroblasts to initiate tissue regenerative responses and fibrosis. J Am Soc Nephrol. 2013; 24(3): 385-392, doi: 10.1681/ASN.2012101031, indexed in Pubmed: 23274427.

31. van den Akker J, van Weert A, Afink G, et al. Transglutaminase 2 is secreted from smooth muscle cells by transamidation-dependent microparticle formation. Amino Acids. 2012; 42(2-3): 961-973, doi: 10.1007/s00726-011-1010-3, indexed in Pubmed: 21830119.

32. Waldenström A, Gennebäck N, Hellman U, et al. Cardiomyocyte microvesicles contain DNA/RNA and convey biological messages to target cells. PLoS One. 2012; 7(4): e34653, doi: 10.1371/journal. pone.0034653, indexed in Pubmed: 22506041.

33. Boon RA, Vickers KC. Intercellular transport of microRNAs. Arterioscler Thromb Vasc Biol. 2013; 33(2): 186-192, doi: 10.1161/ATVBAHA.112.300139, indexed in Pubmed: 23325475.

34. Kervadec A, Bellamy V, El Harane N, et al. Cardiovascular progenitor-derived extracellular vesicles recapitulate the beneficial effects of their parent cells in the treatment of chronic heart failure. J Heart Lung Transplant. 2016; 35(6): 795-807, doi: 10.1016/j.healun.2016.01.013, indexed in Pubmed: 27041495.

35. Wang Y, Zhang L, Li Y, et al. Exosomes/microvesicles from induced pluripotent stem cells deliver cardioprotective miRNAs and prevent cardiomyocyte apoptosis in the ischemic myocardium. Int J Cardiol. 2015; 192: 61-69, doi: 10.1016/j.ijcard.2015.05.020, indexed in Pubmed: 26000464 .

36. Vrijsen KR, Sluijter JPG, Schuchardt MWL, et al. Cardiomyocyte progenitor cell-derived exosomes stimulate migration of endothelia cells. J Cell Mol Med. 2010; 14(5): 1064-1070, doi: 10.1111/j.1582-4 934.2010.01081.x, indexed in Pubmed: 20465578.

37. Mackie AR, Klyachko E, Thorne T, et al. Sonic hedgehog-modified human CD34+ cells preserve cardiac function after acute myocardial infarction. Circ Res. 2012; 111(3): 312-321, doi: 10.1161/CIRCRESAHA.112.266015, indexed in Pubmed: 22581926.

38. Varga ZV, Zvara A, Faragó N, et al. MicroRNAs associated with ischemia-reperfusion injury and cardioprotection by ischemic preand postconditioning: protectomiRs. Am J Physiol Heart Circ Physiol. 2014; 307(2): H216-H227, doi: 10.1152/ajpheart.00812.2013, indexed in Pubmed: 24858849.

39. Li J, Xuan W, Yan R, et al. Remote preconditioning provides potent cardioprotection via PI3K/Akt activation and is associated with nuclear accumulation of $\beta$-catenin. Clin Sci (Lond). 2011; 120(10): 451-462, doi: 10.1042/CS20100466, indexed in Pubmed: 21143191.

40. Wang X, Huang W, Liu G, et al. Cardiomyocytes mediate anti-angiogenesis in type 2 diabetic rats through the exosomal transfer of miR-320 into endothelial cells. J Mol Cell Cardiol. 2014; 74: 139-150, doi: 10.1016/j.yjmcc.2014.05.001, indexed in Pubmed: 24825548.

41. Kawaguchi N, Smith AJ, Waring CD, et al. GATA-4 high rat cardiac stem cells foster adult cardiomyocyte survival through IGF-1 paracrine signalling. PLoS One. 2010; 5(12): e14297, doi: 10.1371/journal.pone.0014297, indexed in Pubmed: 21179204 .

42. Chen MM, Lam A, Abraham JA, et al. CTGF expression is induced by TGF- beta in cardiac fibroblasts and cardiac myocytes: a potential role in heart fibrosis. J Mol Cell Cardiol. 2000; 32(10): 1805-1819, doi: 10.1006/jmcc.2000.1215, indexed in Pubmed: 11013125.

43. Zhang Y, Huang XR, Wei LH, et al. miR-29b as a therapeutic agent for angiotensin II-induced cardiac fibrosis by targeting TGF- $\beta /$ Smad 3 signaling. Mol Ther. 2014; 22(5): 974-985, doi: 10.1038/mt.2014.25, indexed in Pubmed: 24569834.

44. Roy S, Khanna S, Hussain SRA, et al. MicroRNA expression in response to murine myocardial infarction: miR-21 regulates fibroblast metalloprotease-2 via phosphatase and tensin homologue. Cardiovasc Res. 2009; 82(1): 21-29, doi: 10.1093/cvr/cvp015, indexed in Pubmed: 19147652.

45. Lenferink AEG, Cantin C, Nantel A, et al. Transcriptome profiling of a TGF-beta-induced epithelial-to-mesenchymal transition reveals extracellular clusterin as a target for therapeutic antibodies. Oncogene. 2010; 29(6): 831-844, doi: 10.1038/onc.2009.399, indexed in Pubmed: 19935703.

46. Lyu L, Wang H, Li B, et al. A critical role of cardiac fibroblast-derived exosomes in activating renin angiotensin system in cardiomyocytes. J Mol Cell Cardiol. 2015; 89(Pt B): 268-279, doi: 10.1016/j.yjmcc.2015.10.022, indexed in Pubmed: 26497614.

47. Casal E, Palomo L, Cabrera D, et al. A Novel Sensitive Method to Measure Catechol-O-Methyltransferase Activity Unravels the Presence of This Activity in Extracellular Vesicles Released by Rat Hepatocytes. Front Pharmacol. 2016; 7: 501, doi: 10.3389/fphar.2016.00501, indexed in Pubmed: 28066248.

48. Kakimoto Yu, Ito S, Abiru H, et al. Sorbin and SH3 domain-containing protein 2 is released from infarcted heart in the very early phase: proteomic analysis of cardiac tissues from patients. J Am Heart Assoc. 2013; 2(6): e000565, doi: 10.1161/JAHA.113.000565, indexed in Pubmed: 24342996.

49. Bang C, Batkai S, Dangwal S, et al. Cardiac fibroblast-derived microRNA passenger strand-enriched exosomes mediate cardiomyocyte hypertrophy. J Clin Invest. 2014; 124(5): 2136-2146, doi: 10.1172/JCI70577, indexed in Pubmed: 24743145.

50. Queirós AM, Eschen C, Fliegner D, et al. Sex- and estrogen-dependent regulation of a miRNA network in the healthy and hypertrophied heart. Int J Cardiol. 2013; 169(5): 331-338, doi: 10.1016/j. ijcard.2013.09.002, indexed in Pubmed: 24157234.

Cite this article as: Gąsecka, A, van der Pol E, Nieuwland R, et al. Extracellular vesicles in post-infarct ventricular remodelling. Kardiol Pol. 2018; 76(1): 69-76, doi: 10.5603/KP.a2017.0178. 